\title{
Tiempo de reevaluar los bisfosfonatos y de revisar la regulación de medicamentos y nuestras actitudes
}

\section{Time to reassess bisphosphonates, Drug regulations, and rethink our attitudes}

\section{J. Erviti López ${ }^{1}$, J. Gorricho Mendívil ${ }^{2}$}

\section{Sr. Director:}

En el número 36.1 de la revista Anales del Sistema Sanitario de Navarra, OuteiriñoFernández ${ }^{1}$ revisa los factores de riesgo de osteonecrosis de mandíbula asociada al uso de bisfosfonatos. Esta complicación producida por los mencionados medicamentos fue descrita hace ya bastantes años. Parece que este efecto adverso óseo no se localiza específicamente en la mandíbula sino que puede darse en cualquier localización ${ }^{2}$. Uno de los motivos que ha influido en que sea en los maxilares donde primero se ha identificado este problema es que es un hueso sometido a intervención quirúrgica con cierta frecuencia, especialmente por la colocación de implantes dentales.

Realmente sorprende que unos fármacos que supuestamente mejoran la calidad del hueso acaben produciendo una necrosis del mismo, dolor óseo o fracturas de subtrocánter y diáfisis.

Los bisfosfonatos fueron aprobados para su uso en humanos en virtud de su capacidad de incrementar la densidad mineral ósea. Esta observación no era de extrañar por el simple hecho de que se depo- sitan y permanecen en los huesos durante muchos años. Se dio por supuesto que el aumento de la densidad implicaría una mejora en la microarquitectura y en la resistencia ósea. Sin embargo, esta hipótesis no está demostrada.

Algunos años después de la salida al mercado de los bisfosfonatos, se realizaron ensayos que medían sus efectos sobre la reducción de fracturas vertebrales morfométricas radiológicas. Este tipo de "fracturas" se define como la pérdida de altura entre un $20-25 \%$ de una vértebra en cualquier punto de la misma. Se trata de una variable de medida que no es práctica clínica habitual y que se desarrolló de forma concomitante a la realización de los ensayos con alendronato. Los propios autores que la desarrollaron reconocen sus limitaciones en cuanto a la arbitrariedad con la que se puede llegar al diagnóstico ${ }^{3}$. Su relevancia clínica es muy cuestionable. Además, en la mayoría de los ensayos, las fracturas vertebrales clínicas son, aproximadamente, un tercio de las fracturas vertebrales morfométricas radiológicas. Dicho de otra forma, casi el $70 \%$ de las fracturas vertebrales diagnosticadas mediante este método no tienen relevancia clínica.
1. Jefe de la Sección de Información y Asesoría del Medicamento. SNS-O

2. Servicio de Prestaciones Farmacéuticas. SNS-O

\author{
Correspondencia: \\ Juan Erviti López \\ Sección de Información y Asesoría del \\ Medicamento \\ Plaza de la Paz, s/n, planta $4^{\mathrm{a}}$ \\ 31002 Pamplona \\ E-mail: jervitil@navarra.es
}


Cuando analizamos los datos en fracturas de cadera, la situación no es mejor, ni mucho menos. En la mayor parte de los ensayos pivotales con alendronato, risedronato o ibandronato frente a placebo, las fracturas de cadera son variables secundarias y no se observó una reducción en la incidencia asociada al uso de estos fármacos. Se han realizado distintos metanálisis con alendronato y risedronato en los que se concluye que estos medicamentos pueden reducir la incidencia de fracturas de cadera de forma estadísticamente significativa, si bien la magnitud del efecto es muy pequeña. Sin embargo, estos metanálisis cuentan con graves sesgos de diseño que nos hacen cuestionar la validez de sus conclusiones. Cuando se valora la calidad de los ensayos clínicos incluidos en los metanálisis, se puede observar que el $75 \%$ de los ensayos tenían un riesgo de sesgo "dudoso o alto". Esto significa que la supuesta pequeña reducción del riesgo de fracturas de cadera pudiera no ser real ${ }^{4}$.

En 2006 se publicó el ensayo de mayor duración con bisfosfonatos ${ }^{5}$. Después de 5 años en tratamiento con alendronato, se aleatorizó a las mujeres a seguir otros 5 años en tratamiento con el fármaco o a recibir placebo durante ese tiempo. Se observó que la suspensión del tratamiento de alendronato no produjo un aumento de fracturas cinco años después. Por tanto, la prolongación del tratamiento no aportaba ningún beneficio. Por otro lado, todas las pacientes habían tomado el fármaco durante 5 años, por lo que no se pueden establecer conclusiones respecto a las mujeres que nunca recibieron el fármaco.

En un artículo publicado por investigadores de la FDA, se analizaron los resultados de la extensión de tres ensayos clínicos con bisfosfonatos (alendronato, risedronato y zoledrónico). Los datos conjuntos de estos estudios revelaron que el uso de bisfosfonatos durante 6 años o más se asociaba con unas incidencias de fracturas del 9,3 al $10,6 \%$, mientras que los pacientes que pasaron a recibir placebo tenían incidencias entre 8,0 y $8,8 \%$. Los propios revisores de la FDA se cuestionan si el uso de bisfosfonatos es beneficioso a largo plazo ${ }^{6}$.
En 2008 se realizó un estudio de cohortes con una base de datos poblacional de Dinamarca. Se observó que la incidencia de fracturas de cadera en el grupo que recibió alendronato se incrementaba en un $50 \%$ en términos relativos y en 6 casos por 1000 mujeres-año en términos absolutos ${ }^{7}$. En 2010 se publicó información actualizada de este estudio que confirmó los resultados obtenidos anteriormente ${ }^{8}$.

En 2013 se publicó un estudio de casos y controles anidado en una cohorte española en el que no se observó eficacia alguna de los bisfosfonatos en la prevención de fractura de cadera en la práctica clínica. $\mathrm{Al}$ valorar el tiempo transcurrido desde el comienzo del tratamiento, se vio que las mujeres tratadas con bisfosfonatos incrementaban su riesgo de fractura de cadera a partir del tercer año respecto a las que nunca tomaron estos fármacos ${ }^{9}$. Por otro lado, se pudo constatar en la misma población que el uso de bisfosfonatos se asocia con un importante incremento del riesgo de fracturas de subtrocánter y diáfisis, OR (IC 95\%) $=4,30(1,55 \text { a } 11,9)^{10}$.

Se han identificado los mecanismos de acción que explican los efectos óseos nocivos producidos por los bisfosfonatos. Son medicamentos que inhiben el recambio óseo, lo que debilita la estructura del hueso. Además, aumenta la mineralización, lo que hace perder resistencia y evolucionar hacia huesos de cristal (brittle bones $)^{11}$.

Con una evidencia tan cuestionable sobre los supuestos beneficios óseos de los bisfosfonatos, nos hemos lanzado de una forma poco reflexiva a tratar masivamente a las mujeres. Ya hay precedentes al respecto, como es el uso generalizado de terapia hormonal sustitutiva en mujeres postmenopáusicas. Cuando se realizó el primer ensayo clínico adecuado para evaluar los efectos en variables clínicamente relevantes, se observó un incremento del riesgo de enfermedad coronaria e ictus, entre otros efectos indeseados, que condicionó el abandono generalizado de esta práctica ${ }^{12}$. En gran medida, la prescripción de terapia hormonal fue sustituida por bisfosfonatos, nuevamente sin tener datos suficientes de 
que esta intervención produjera unos claros beneficios en la salud de las mujeres.

El caso de los bisfosfonatos muestra, una vez más, que las agencias reguladoras aprueban medicamentos sin datos convincentes de eficacia en variables clínicamente relevantes. Además, reaccionan con gran lentitud ante las señales de seguridad importantes y tienen demasiadas dificultades para tomar la decisión de retirar medicamentos de beneficio-riesgo cuestionable o desfavorable, como se ha visto recientemente con el caso de las calcitoninas.

Lo que no cabe ninguna duda es que las campañas promocionales son muy eficaces y responden a un guión preestablecido que pivota sobre tres ejes: exagerar la prevalencia y la importancia de la enfermedad o factor de riesgo, promover el diagnóstico y magnificar la eficacia y seguridad de los tratamientos.

Con respecto al diagnóstico, hay que considerar que la baja densidad mineral ósea (DMO) es un factor de riesgo de fracturas. Sin embargo, las pruebas de densitometría ósea presentan importantes limitaciones y no son útiles para realizar programas de cribado ${ }^{13}$. El límite de definición de osteoporosis (DMO <-2,5 DE) es arbitrario y los expertos que lo determinaron reconocieron que el dato fue fruto de un consenso y no basado en evidencias científicas.

Por si esto fuera poco, se propuso el concepto de osteopenia, entendido como aquellas personas con una DMO entre -1 y $-2,5 \mathrm{DE}$, y hay quien propugna tratar farmacológicamente a estas personas sin dato alguno que lo avale. Dicho en otras palabras, se ha conseguido etiquetar como "enferma" a una población muy grande y sana.

La promoción del diagnóstico cuenta con la colaboración de sociedades científicas y "expertos" que, en ocasiones, con intereses económicos de por medio, facilitan la elaboración de guías, protocolos, campañas de sensibilización como el "día mundial de la osteoporosis", cribados, etc., cuyo fin último es etiquetar de osteoporóticas al mayor número posible de personas sanas. El papel de muchos medios de comunicación también es muy relevante y, en ocasiones, se ofrecen a alimentar todas estas estrategias.

También debe mencionarse que nuestra sociedad es un buen caldo de cultivo para la medicalización irreflexiva. Pensamos que la medicina lo tiene que solucionar todo con una pastilla. Realmente es una situación dura cuando un paciente sufre un deterioro limitante en relación con un proceso de osteoporosis. Sin embargo, debemos ser honrados y reconocer que la medicina no lo puede arreglar todo y que, hoy en día, desgraciadamente los medicamentos no son una buena alternativa para los problemas asociados a la osteoporosis.

Nuestra sociedad necesita una serie de cambios importantes. En primer lugar, se requiere que las agencias reguladoras de medicamentos sean transparentes e independientes y que defiendan los intereses de los pacientes por encima de los intereses de mercado.

Es necesario también potenciar la investigación independiente de la industria farmacéutica. En estos tiempos en los que se aprueban medicamentos con muy pocos datos y de forma acelerada, se hace imprescindible la farmacoepidemiología como forma de evaluar los efectos reales de los medicamentos en la práctica clínica habitual.

Por otro lado, es fundamental que las guías de práctica clínica y sociedades científicas no estén influenciadas por la industria y se orienten a proponer las mejores soluciones para los pacientes, fundamentadas en una lectura crítica y responsable de la evidencia científica disponible. Respecto a los enfermos, también es importante que cuenten con una buena información y que las sociedades de pacientes no estén contaminadas por la industria farmacéutica dado que, a la larga, tanto los enfermos como la sociedad en general salen malparados de esta relación.

Y, por último, necesitamos políticos que realmente crean que las personas son más importantes que el mercado.

En España hemos invertido ingentes recursos humanos y económicos en el diagnóstico y tratamiento de la osteoporosis. No está nada claro que el uso masivo de 
bisfosfonatos haya aportado algo positivo y sí que tienen su alto coste económico y efectos indeseables. Desgraciadamente, el resto de fármacos para tratar este factor de riesgo no son mejores. Recientemente se han retirado las calcitoninas, tras más de 40 años en el mercado, debido a su ligero incremento del riesgo de cáncer y su cuestionable eficacia en la prevención de fracturas ${ }^{14}$. ¿Ocurrirá algo parecido con los bisfosfonatos? ¿Aprenderemos alguna vez algo de todas estas historias?

\section{BIBLIOGRAFÍA}

1. Outeriño-Fernández, A. La osteonecrosis asociada al tratamiento con bisfosfonatos en osteoporosis primaria. Revisión de la literatura. An Sist Sanit Navar 2013: 36: 87-97

2. Vlad SC, Zhu Y, Niu J, et al. Bisphosphonate use may be a risk factor for osteonecrosis at any site: a self-controlled case series analysis [abstract]. 27th ICPE: International Conference on Pharmacoepidemiology \& Therapeutic Risk Management. Chicago, $2011 ;[274]$

3. Genant HK, Jergas M, Palermo L, Nevitt M, San Valentin R, Black D et al. Comparison of Semiquantitative Visual and Quantitative Morphometric Assessment of Prevalent and Incident Vertebral Fractures in Osteoporosis. J Bone and Mineral Research 1996; 11 : 984-996.

4. Therapeutics initiative. A systematic review of the efficacy of bisphosphonates. Therapeutics Letter 2011; 83: 1-2.

5. Black DM, Schwartz AV, Ensrud KE, Cauley JA, Levis S, Quandt SA et al. Effects of continuing or stopping alendronate after 5 years of treatment. The fracture intervention trial long-term extension (FLEX): a randomized trial. JAMA 2006; 296: 2927-2938.

6. Whitaker M, Guo J, Kehoe T, Benson G. Bisphosphonates for osteoporosis. Where do we go from here? N Engl J Med 2012; May 9, 2012 (10.1056/NEJMp1202619)
7. Abrahamsen B, Eiken P, Eastell R. Subtrochanteric and diaphyseal femur fractures in patients treated with alendronate: a register-based national cohort study. JBMR, doi: 10.1359/JBMR.081247.

8. Abrahamsen B, Eiken P, and Eastell R. Cumulative alendronate dose and the long-term absolute risk of subtrochanteric and diaphyseal femur fractures: A register-based national cohort analysis. J Clin Endocrin Metab doi:10.1210/jc.2010-1571.

9. Erviti J, Alonso Á, Gorricho J et al. Oral bisphosphonates may not decrease hip fracture risk in elderly Spanish women: a nested case-control study. BMJ Open 2013; 3:e002084. doi:10.1136/bmjopen-2012-002084

10. Erviti J, Alonso Á, Oliva B, et al. Oral bisphosphonates are associated with increased risk of subtrochanteric and diaphyseal fractures in elderly women: a nested case-control study. BMJ Open 2013;3:e002091. doi:10.1136/ bmjopen-2012-002091

11. Odvina CV, Zerwekh JE, Rao DS, Maalouf N, Gottschalk FA, Pak CY. Severely suppressed bone turnover: a potential complication of alendronate therapy. J Clin Endocrinol Metab 2005; 90: 1294-1301.

12. Writing Group for the Women's Health Initiative Investigators. Risks and Benefits of Estrogen Plus Progestin in Healthy Postmenopausal Women: Principal Results From the Women's Health Initiative Randomized Controlled Trial. JAMA. 2002; 288:321-333. doi:10.1001/jama.288.3.321.

13. Marshall D, Johnell O, Wedel H. Meta-analysis of how well measures of bone mineral density predict occurrence of osteoporotic fractures. BMJ 1996; 312: 1254.

14. Agencia Española de Medicamentos y Productos Sanitarios AEMPS. Calcitonina: suspensión de la comercialización de los preparados intranasales y restricción del uso de los preparados inyectables a tratamientos de corta duración. Disponible en: http://www. aemps.gob.es/informa/notasInformativas/ medicamentosUsoHumano/seguridad/2013/ NI-MUH_FV_09-2013-calcitonina.htm 